\title{
Challenges in Sensors Technology for Industry 4.0 for Futuristic Metrological Applications
}

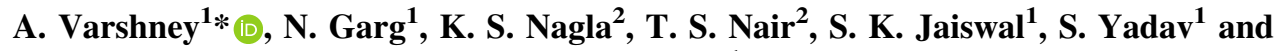 \\ D. K. Aswal ${ }^{1}$ \\ ${ }^{1}$ CSIR-National Physical Laboratory, New Delhi 110 012, India \\ ${ }^{2}$ Dr. B R Ambedkar National Institute of Technology, Jalandhar 144 011, India
}

Received: 28 March 2021 / Accepted: 12 April 2021 / Published online: 3 May 2021

(C) Metrology Society of India 2021

\begin{abstract}
The current advances and innovations in sensor technologies attributed to Industry 4.0 serve as the backbone for the inclusive growth of industry and ramping up the economy of any country. Industry 4.0 was basically conceptualized by introducing the Internet of Things (IoT) and Information and Communication technologies (ICT) that serve as an interface between digital and physical world through the fusion of smart sensors. The role of smart sensors and IoT-enabled industrial infrastructure is pivotal for adapting to the advanced technologies based on fusion of smart sensors. Digital meteorological traceability and uses of intelligent sensors, instrumentation and machinery in Industry 4.0, Smart Cities, Digital India and AtmaNirbhar Bharat missions of the government of India, are not only highly important but also in huge demand, which is going to increase manifolds in the years to come. The present paper is an attempt to provide a terse review and perspectives related to the advanced technological developments in this field and the challenges therein.
\end{abstract}

Keywords: Smart sensor; Industry 4.0; Internet of Things (IoT); Information and communication technology (ICT); Cyber physical systems; Digital metrology

\section{Introduction}

Industry 4.0 is playing a vital role in the digital transformation by integrating information and communication technologies (ICT), cyber physical systems, cloud computing, data exchange, retrieval, storage and security framework in the industrial and manufacturing units in recent times. In the twenty-first century, society is moving fast towards ample digital transformation, and it has become the main constituent of the development of digital quality infrastructure (DQI). The process of generation, measurement and transfer of accurate and precise data traceable to the international standards is rising in any digital economy. Digital transformation is currently in the development stage though Industry 4.0 was introduced several years back [1]. The digitalization mainly depends upon the following constituents, (i) computation facilities and storage capability, (ii) speed of data transfer, (iii) costeffectiveness and availability of smart sensors, (iv) proper

*Corresponding author, E-mail: varshney.anshul@nplindia.org use of the stored data and information, (v) development of cyber physical systems, etc. [2-4]. In Industry 4.0, various types of smart sensors are integrated that are used to measure and process quality parameters. These sensors process/transfer data by means of electronic medium or wireless signal [5]. However, for maintaining the highest levels of accuracy, calibration of smart sensors with respect to some digital or electronic standards is required. The redefinition and implications of SI units also provides motivation for the development of advanced reference standards with lowest levels of measurement uncertainty for calibrating these smart instruments. The Internet of Things (IoT) is more frequently applied in metrology for calibration and testing purpose. This is how Industry 4.0 enables the coordination and interaction among workers and machines through cyber physical systems and ICT [6, 7]. Security of measurements and calibration data is the main concern while establishing the metrological infrastructure framework in industrial applications. This creates a need for integrating IoT with the manufacturing processes and systems that are responsible for optimizing the performance of industry and creating the sustainable cyber secure 
system [4]. The digital transformation of manufacturing processes enhances the reliability, security, competitiveness, and transparency of the measured data over smart sensors [8]. There had been many research programmes and collaborative projects carried out in international scenario focussed towards the digital transformation and innovative solutions such as the establishment of metrology cloud, 'NIST on a chip [9], Measurement Information Infrastructure (MII) for various metrological and industrial applications, which is only possible through integration of smart sensors and visualizing the concept of Industry 4.0 [10]. In particular, the prime consideration of such technological advancements is to facilitate the machine readability, storage and transmissibility of the measured data over a distance $[10,11]$.

The metrological framework for digitalization utilizing the smart sensor networks is currently at primitive stages at various National Metrological Institutes (NMIs) across the globe. Metrology, standardization, calibration, testing, accreditation and legal metrology are the main pillars of establishment of a strong National Quality Infrastructure (NQI) in any country. Of late, the 'Aswal Model', developed for robust NQI, also sturdily advocates the transformation towards digital SI and safe and secure certification system, keeping metrology centric and connecting all other pillars of the NQI, for enhancing the inclusive economic growth of the country [12-14]. Thus, establishment of a strong NQI in conjunction with embedded IoT and smart sensor framework would be instrumental in optimizing the production efficiency, improving process and quality parameters for industrial growth and avoiding faults at an early stage [15]. The present work thus focuses on the various aspects in Indian perspectives as follows:

- Relevance and importance of the advanced technologies pertaining to the development of smart sensors network in Industry 4.0 for augmenting the economic growth,

- Digital transformation of measurement process through fusion of smart sensors and IoT-embedded systems that can be very instrumental in accomplishing standardization, metrology accreditation and conformity assessment services so that products and services meet the relevant national and international standards and various goals [16-22],

- The applicability of smart sensors in Industry 4.0 and its implications in metrology 4.0 along with digital transformation of metrological applications with inclusion of smart sensors [17-20],

- Establishment of a national digital quality infrastructure (NDQI) in next few years based on the development of Measurement Information Infrastructure (MII), metrology cloud, digital calibration certificate and integration of advanced technological infrastructure as focussed in European and American continent.

The legally relevant measurements in some of the developed economies are responsible for higher industrial and economic growth. As per an estimate, a share of $4 \%$ to $6 \%$ of the European gross domestic product (GDP), equivalent to 660-990 billion Euros per year, is achieved mainly because of such legal measurements [22]. It is envisaged that if these aspects are carefully planned, devised and implemented in Indian scenario, such an arrangement would be very effective in increasing the GDP in next few years and support the Industry 4.0, Smart cities, Digital India and AtmaNirbhar Bharat missions of the government of India and accomplish the target of achieving USD 5 trillion economy status.

This paper is organized as follows: Introduction to Industry 4.0, its relevance and implications are illustrated in section II. Section II presents the role of smart sensors in Industry 4.0 and concept of digitalization in Industry 4.0. Section III elaborates the impact of Industry 4.0 on metrology and presents the concept of digitalization in metrology along with implementation of metrology cloud and digital quality infrastructure. The various changes and way forward in sensor technologies and digital transformation are illustrated in section IV followed by the concluding remarks in section $\mathrm{V}$.

\section{Industry 4.0: Relevance and Implications}

The first industrial revolution, i.e. Industry 1.0, started in the eighteenth century. In Industry 1.0, manual labourbased industry towards the use of steam engines, etc., to increase the productivity was practised. This was also called mechanization of industries. Industry 2.0 started after a century with the utilization of electricity and assembly line productions. Further, Industry 3.0 was introduced with the advent of the memory-programmable computers and electronics control and came into mainframe in the twentieth century to boost the production process and product standardization. In recent times, automation has become the game-changing factor for manufacturing and process industries. The human interaction with the machines is reduced in manufacturing processes by the inclusion of digital process control and automation [23]. The fourth industrial revolution, Industry 4.0, was initiated by the advanced and latest technological innovations which brought the cyber physical systems together, i.e. smart machines capable of exchanging realtime information over the Industrial Internet of Things (IIoT) for decision-making process. 
The Industry 4.0 is known as the fourth industrial revolution, which represents a new industrial infrastructure based on cyber physical systems having embedded software connected through IoT to the physical word. Thus, this framework facilitates the easy and faster communication in the production process by creating a real-time optimization, modern control systems and enabling predictive maintenance by introducing machine learning (ML) and artificial intelligence (AI) component [24].

The improvements in cyber computing facilities are the main factor behind the popularity and reliability of Industry 4.0. These advanced technologies are responsible for narrowing the gap between virtual and real world by creating an interface between ICT and actual operations. The basic structure of Industry 4.0 is shown in Fig. 1, and timeline of industrial revolution is shown in Fig. 2 [4].

These types of industries, containing all these smart peripherals, are also known as Factory of Future (FoF). The capabilities required for FoF, like remote monitoring, fault tracking, remote sensing, cloud computing, data analytics and cognitive computing, etc., are created by the cyber physical systems. These smart industries are equipped with smart sensors, intelligent automation techniques and equipment, which can collect and analyse data and allow for better decision making. Moreover, intelligent networking among different platforms enables communication between machines and manufacturing process parameters that are developed by using IoT and ICT [25].

Manufacturing, construction, shipping and supply chain management sectors are directly being influenced by Industry 4.0, which directly impacts on day-to-day activities. The concept of Industry 4.0 refers to the smart industry or cyber industry with digitalization of all the industrial processes, characterized by its reliability,

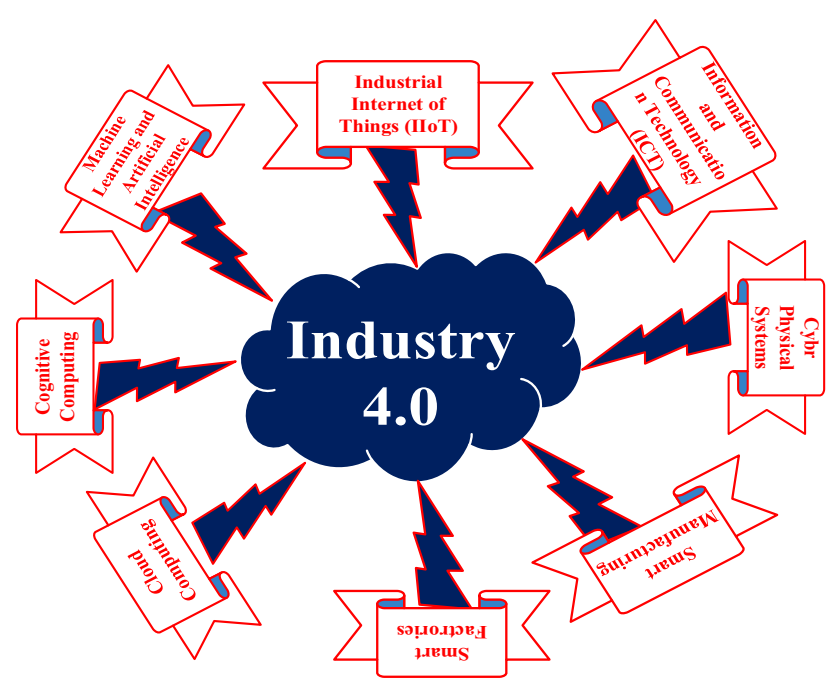

Fig. 1 Major building blocks of Industry 4.0 flexibility and adaptability, which also take care of the consumer's need in the market [26, 27]. Eventually, the major benefits are as $[8,28]$ :

- Enhancement in productivity based on real information.

- Efficient utilization of resources.

- Real-time optimization of productive processes

- Increased flexibility for controlling the processes.

- Establishes direct link between organizations and users.

- Minimization of down time based on predictive fault analysis.

- Product standardization and quality assurance

\subsection{Role of Smart Sensors in Industry 4.0}

The key components of Industry 4.0 are the industrial internet of things, which includes different types of sensors for controlling and monitoring the process parameters $[17,29]$. These sensors relate to the process control equipment either remotely or physically. The role of smart sensors in Industry 4.0 is summarized in Fig. 3. Smart sensors and actuators are the first levels of process automation. Basically, automation structure consists of different stages that has been classified as [30]:

Stage 1-This stage consists of sensors and actuators, which collects raw data from manufacturing process and initiates the control by analysing that data.

Stage 2-This stage acts as an interface between process parameters and control parameters by means of various input/output modules.

Stage 3-This stage is known as controlling stage that consists of IT infrastructure, responsible for controlling the production processes.

Stage 4-The function of this stage is monitoring the whole manufacturing process as well as it consists of process management system.

Stage 5-This stage serves as interface consisting of detailed operational planning, quality specification management and data collection over the cloud.

In Industry 4.0, all the machines in the process plant are integrated with sensors having an IP address that allows them to communicate with other IoT-enabled devices. IoTenabled devices make it possible for exchanging a large amount of data for a short span, to optimize and evaluate the performance of the whole system. Moreover, the future prediction, warnings, status updates, etc., about the behaviour of the machines are easily obtained from the analysis of collected data through the sensors [31]. The measurement data collected through the sensors, process control algorithms, mathematical analysis with cyber secure infrastructure is the basis of digital transformation, which makes the quality infrastructure stronger with conformity 
Fig. 2 Timeline of industrial revolution

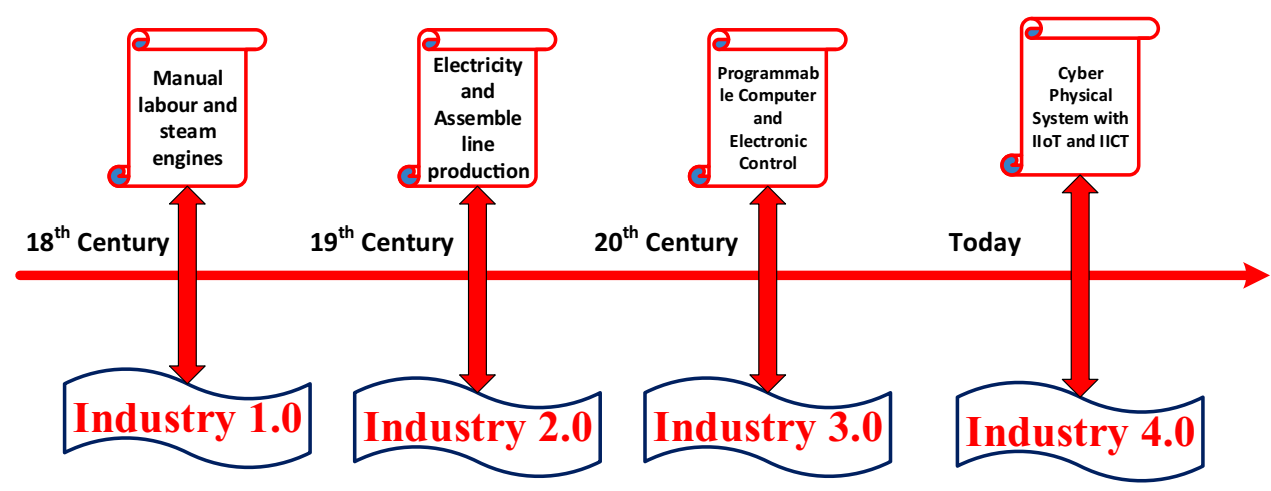

assessment market surveillance and verification system [32]. With the advancements in technologies and development of high-speed computerized software and hardware frameworks, the sensor technology has outgrown and has entered in a hi-tech stage, resulting in precision manufacturing technology with an objective of enhancing the operation monitoring capabilities and accuracy with increasing the efficiency of the process by creating a virtual link between the machines and end users. In the production system, each sensor collects some certain parameters and then the system uses signal processing algorithms and intelligent control techniques to relate all the measurement results in a comprehensive set. These sensors are directly connected to the measurement system or they collect and transfer the data through wireless signal $[33,34]$.

The smart sensors, which are being used in Industry 4.0 for monitoring, measurement and control prospective, need precise calibration methods and standards for ensuring traceability to the national standards and accomplishing the highest levels of accuracy and precision and conformance to relevant national and international standards. Moreover, wireless or remote calibration is preferred for these smart sensors. The past standards which are being adopted for the calibration may not be completely suitable for smart sensor. Thus, the development of advanced calibration standards and methods integrated with IoT-embedded systems and machine readability for Industry 4.0 is the necessity for making the measurements highly accurate, precise and reliable [35]. The manufacturing process has been revolutionized by fourth industrial revolution enabling self-sufficient manufacturing process by using machines and sensors, which can establish a communication link through digital connectivity [2]. A smart factory is characterized with the help of (i) sensors which are having the capability to self-learn on the basis of environmental information and they can take part in the decision-making process according to the change in process variables, (ii) co-ordination between different devices through flexible interconnection, (iii) artificial intelligence (AI) allow smart factories to have an advance level of interconnection among various processes. Moreover, AI with human capabilities allows industries to take the decisions based on the analysis of data and (iv) the most important component of Industry 4.0, which enables human-machine interface by using signal processing techniques and simulating different conditions based on sensor data [31].

\subsection{Implications of Industry 4.0 and Digitalization}

India had become the fastest growing country exceeding China in 2019. But due to the novel coronavirus and lockdown, the GDP of India has seen its lowest peak of the last decade in 2020. This determines the need of digital transformation of the industries in India. Making use of the best IT as well as intellectual resources available India should stride forward to develop the country in shoulder with the global leaders. In 2019-2020 survey of GDP, the contribution of industry sector of India is only $23 \%$, which is $30 \%$ lower than the world's average [36]. Digital users in India are growing at a faster rate. Indians spend more time in social media than any other country except china. The three elements that define digital adoption index are:

Digital foundation (cost, speed and reliability of internet service),

Digital reach (number of mobile devices, app downloads and data consumption), and

Digital value (how much consumers engage online by chatting, tweeting, shopping or streaming).

Figure 4 indicates the comparison of the digital adoption index of various countries [37]. As the citizen of India is growing accustomed to the digital world, it paves the brick for the conversion of conventional industrial services towards the digital industrial services. 
Fig. 3 Major paradigms of Industry 4.0 and smart sensors

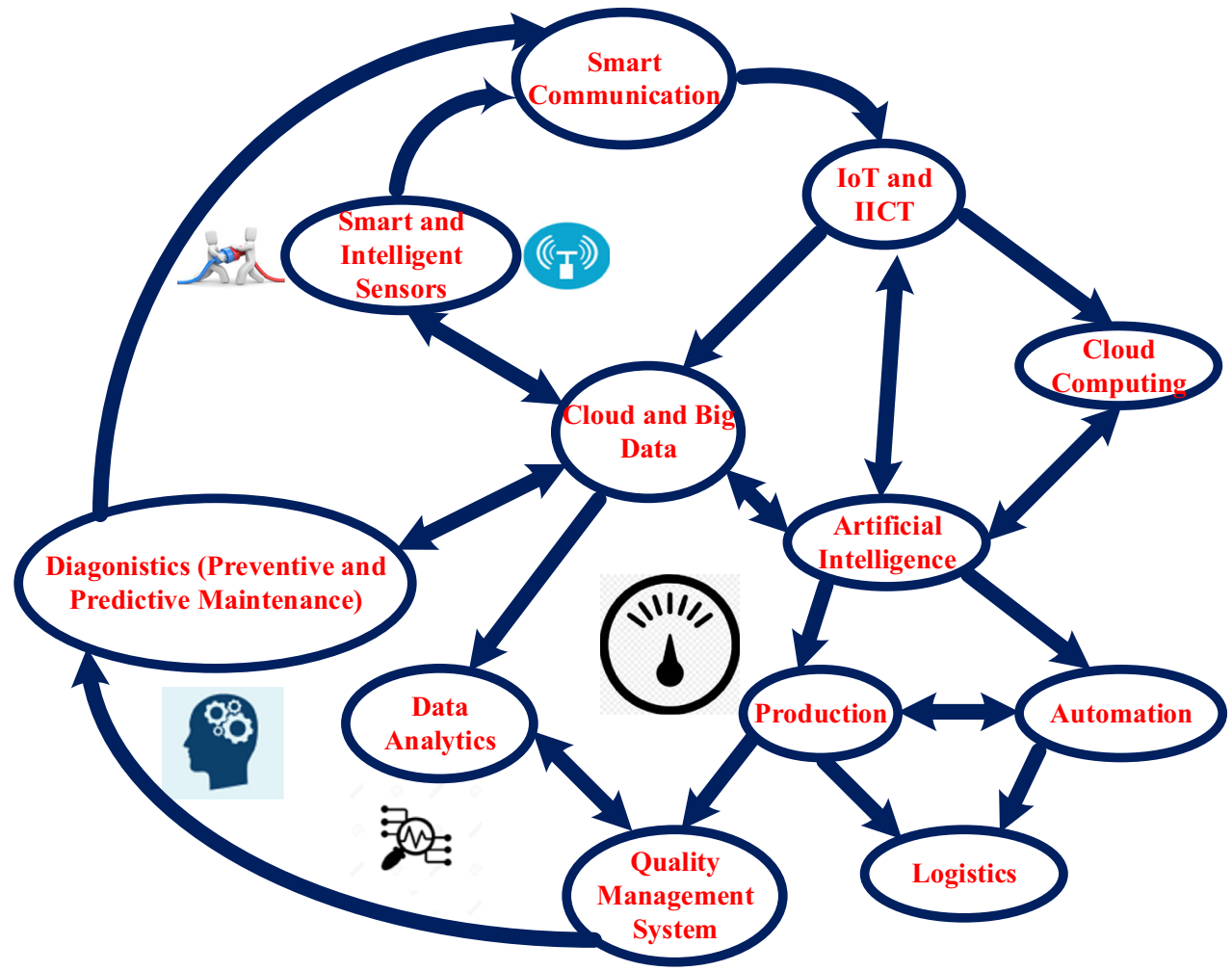

\section{Metrology and Impact of Industry 4.0}

The development of Industry 4.0 concept in turn inculcates challenges and strengthening of the metrological framework also. Thus, it is required for rethinking about the measurement phenomena that can synchronize the Industry 4.0 along with metrology 4.0. There is a huge requirement of online metrology for futuristic industrial requirement. The main goal of Industry 4.0 is to maximize the efficiency and increase the quality of the throughput [38]. The main challenges in metrology are the measurement of multi-dimensional quantities with security of measurement results in real-time mode. The prime consideration is the development of advanced national and international standards regulating the current and futuristic requirement of the industry. Moreover, the implementation of suitable measurement methods and processes conforming to relevant national and international standards along with selection of appropriate equipment is the necessity of Industry 4.0 [39].

\subsection{Digital Transformation in Metrology}

Metrology is the experimental and theoretical evaluation of any uncertainty in the measurement in the field of science and technology. There are three types of metrology, scientific metrology that deals with the measurement standards, industrial metrology that deals with the measurements in an industry and legal metrology dealing with the law and protection of the users of measuring instruments [40]. The major components of metrological infrastructure are the traceability of calibration and analysis of uncertainty with international standards guidelines. Digital transformation of industries and inclusion of data analytics are changing the aspects of measurement with the implication of smart sensors integrated with machine learning algorithms for automated decision making. The objective of enhancing the metrological infrastructure required for improved quality infrastructure in smart factories by adopting traceable calibrations in accordance with the relevant national and international standards developed according to the need of factory of future [41, 42]. The establishment of a virtual linkage between the calibration laboratory and client locations under the umbrella of Industry 4.0 shall be indispensable in calibration of the various sensors in much less time without dismantling them in the system, thus enhancing the accuracy, precision and standardization of the products and services [38]. In this scenario, calibration can be done online/remotely by means of data transport in spite of instrument transport, and data can be transferred from even the apex level body like National Metrology Institutes (NMIs) to client locations electronically. This will result in the saving of time and 
Fig. 4 The comparison of the digital adoption index of various countries [34]

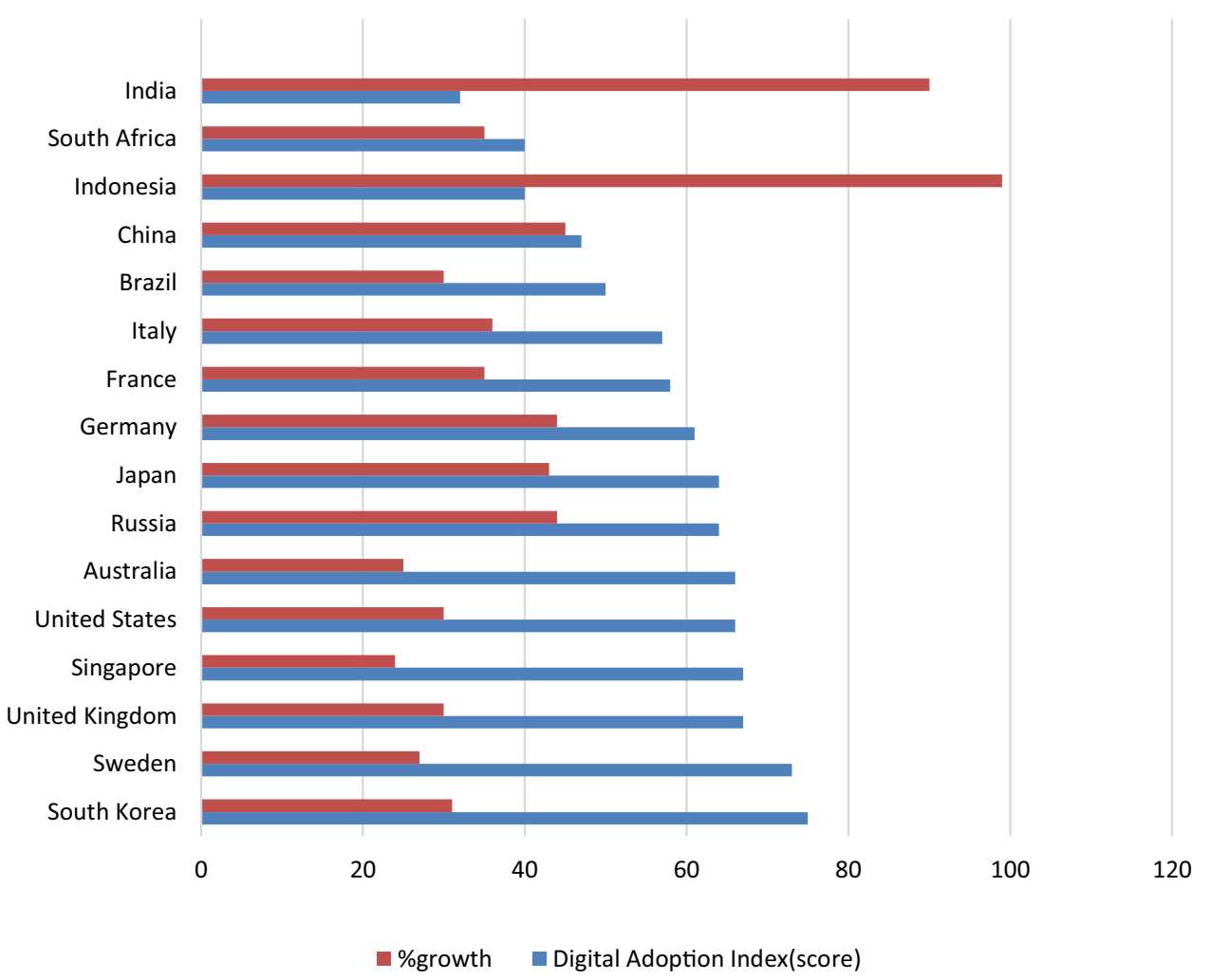

cost without compromising the accuracy/specification of the instruments. The use of sensors is not limited to industry but also influences smart cities and smart mobilities. These sensors are self-tested and calibrated, and its status depending upon its type and method of calibration is recorded. This information is stored in calibration certificates. In Industry 4.0, smart sensors are used that can communicate using wireless communication protocols, so that the calibration values could be communicated to distant places without much manual efforts. Since the sensors today are mostly an IoT device and this measurement can be connected to the cloud services to form an IoMT"Internet of Measurement Things". The architecture of IoMT suggested in reference [43] has three layers integrated-the physical calibration system, Metrological Information Infrastructure Cloud and an application software that can be used in metrology.

Figure 5 describes the major technical attributes of digital transformation in metrology. Kuster et al. 2018 [44] define the novel concept of MII as: A Measurement Information Infrastructure is a set of normative standards that unambiguously define data structures, taxonomies, service protocols and security for locating, communicating and sharing the measurement information. The three primary vehicles for establishment of such an infrastructure are: instrument specifications, statements of accreditations (SOAs) and test and calibration certificates. The involvement of IoT in MII taxonomy establishment can bring synergy to the complete establishment. Also, some innovative measures such as that followed by some NMIs like PTB, Germany, on representing the metrological vocabularies as machine readable metadata with persistent identifier shall be of great support to such an infrastructure [45].

\subsection{Metrology Cloud and Digital Quality Infrastructure}

The establishment of the novel concept of European Metrology Cloud and European Centre of Excellence as highlighted by Thiel F et al., 2017 [22] in order to support the conformity assessment process, market surveillance, data-driven services and reference architectures shall foster the establishment of digital single market and shall cater to the needs of all the stakeholders. The concept of European centre of excellence for metrological information technology comprising of all the experts from the various sectors shall enhance communication, transparency and harmonization and support product standardization, quality assurance and innovations. So, to impose the above tasks a digital quality infrastructure shall be established that will standardize metrology and provide accreditation and conformity assessment. These efforts are also required to be focussed on Indian perspectives so as to establish a national 


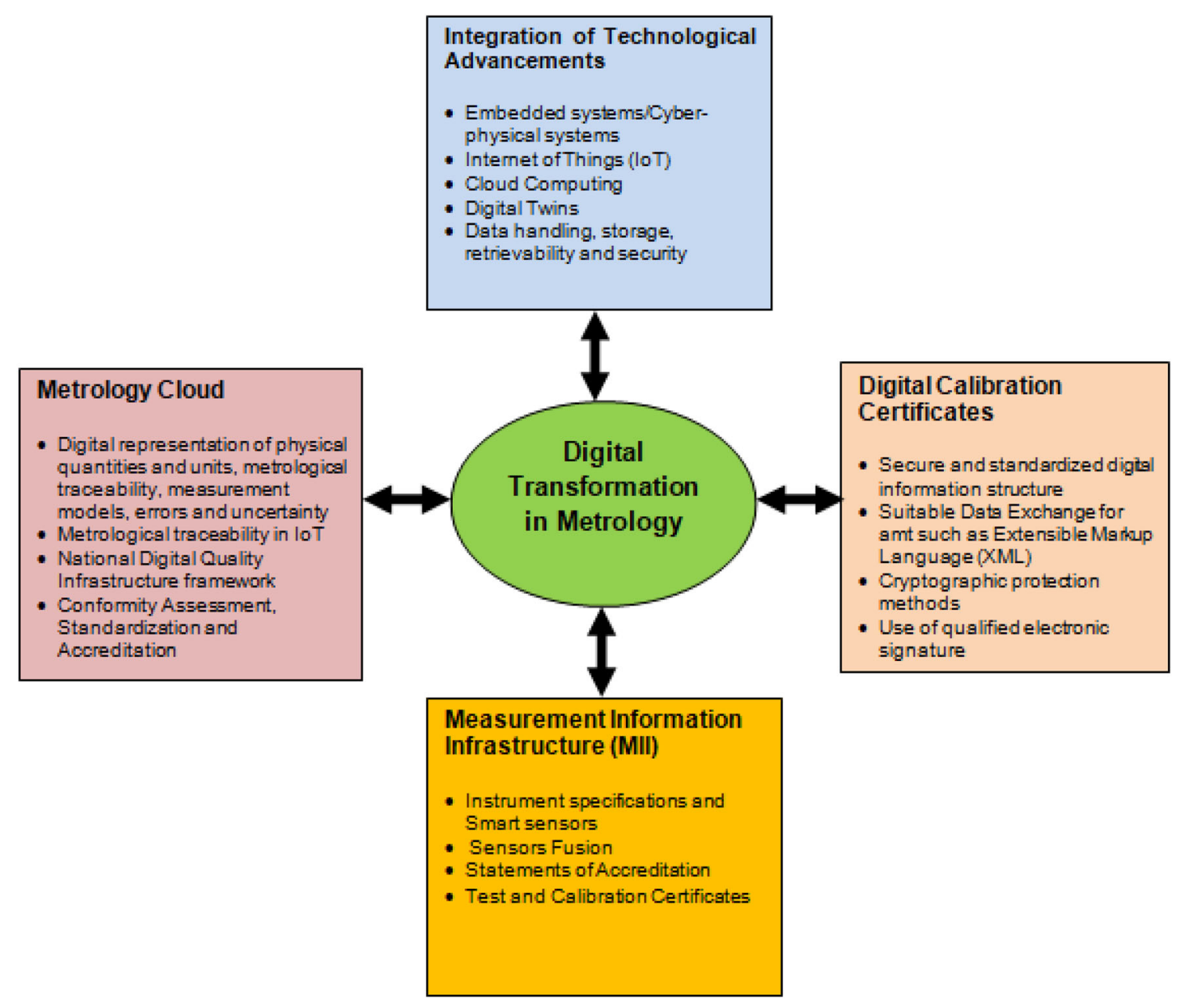

Fig. 5 Major technical attributes of digital transformation in metrology

digital quality infrastructure for supporting the manufacturers and industries and all the stakeholders. Eventually, the role and responsibility of CSIR-National Physical Laboratory, the National Metrology Institute of the India is increased manifold. However, the special budgetary allocations, cooperation of the various stakeholders including experts from industries, manufacturing sector, accreditation bodies, information technology sector, legal metrology and regional laboratories are must. Figure 6 shows the pictorial view of the conceptualized metrology cloud in Indian perspectives on a similar line as that suggested in European Union [22].

The use and implementation of the advanced technologies like IoT framework, cyber physical systems, cloud computing, digital twins shall be very significant in the development of a metrology cloud benefitting all the stakeholders. A digital quality infrastructure integrates the IT infrastructure and databases to the Metrology Grid with a trustworthy connection that incorporates services as well as communication with the data storage. The services may be realized through a concept called "Digital Twin". Digital Twin is a concept used in variety of sectors where a model of a physical instrument with real data is used to assess the performance of a real instrument. The difference of digital twin from a model is that there exists a real physical instrument corresponding to digital twin. There are many definitions for digital twin in the literature. For instance, the LMS Research [46] define it as: "Digital Twin is an executable virtual model of a physical thing or system". As the time changes the model of digital twin also changes. These changes would imitate the defects and faults of the instrument in the real world as the time changes. The digital twin can provide the estimates of and identifies method of minimizing these uncertainties [47]. In Europe, the legal framework is data-driven making use of the European Metrology Cloud to focus on the conformity assessment and market surveillance [22, 48]. The digital single market can provide economic benefits taking the advantage of cloud computing services [49]. A metrological data lake is established to investigate the outcome of 


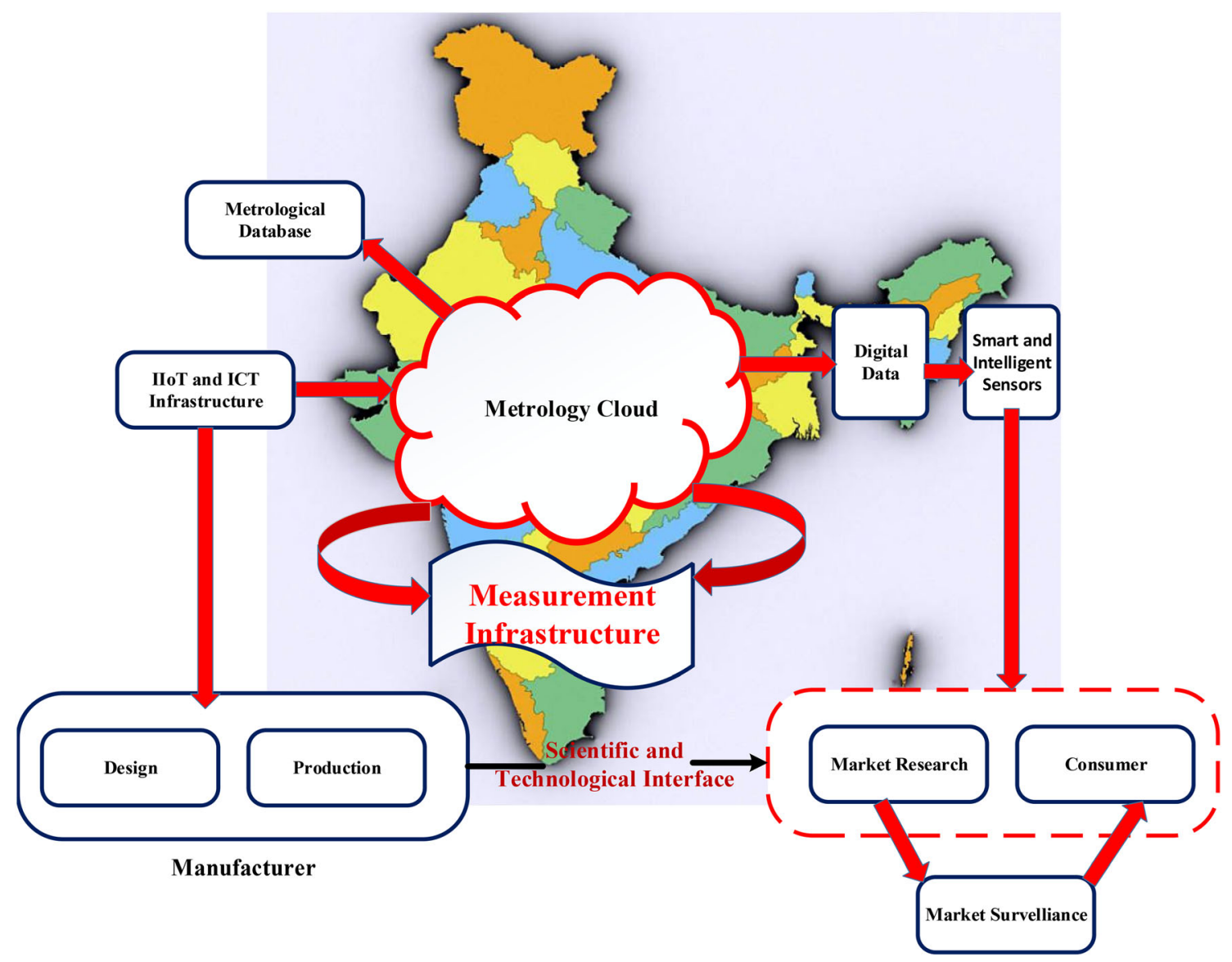

Fig. 6 Conceptualized metrology cloud in Indian perspectives

the combined data from different sources. The outcome of applying the soft computing techniques such as data mining and machine learning on the combine data needs further subtle investigations. The data are obtained from different sources such as the experiences from manufacturers of instruments, from market surveillance and from database itself provide a reliable system that can assess the risk between design and market and transparency provides access to these data for all the stakeholders. Thus, the establishment of such cloud shall strengthen in the development of quality infrastructure and legal metrology that provides a legal framework to protect the interest of a common citizen of India by protecting consumers and instrument users. This can lead to more business opportunities and will stimulate the research and innovation on data-driven technology.

\subsection{Edge Computing in Industry 4.0}

Cyber physical system embeds computational elements connected to physical systems. It forms the pillar for IIoT or Industry 4.0. Various devices in industries such as sensors, servers, mobile robots, workstations are integrated to work together efficiently in a cyber physical system (CPS). The system should be able to communicate within itself and also send data through networking [50]. It is considered that the acquisition and storing of large amount of data in cloud can provide better performance of industry. But the storage of unnecessary data in cloud demands for more storage; hence, the cost increases nulling the benefit of digitalization [51]. The raw data should be filtered so that only required data are stored in the cloud. Edge computing or fog computing stores the data and processes them very fast, thus improving the quality of service and experience [52]. The involvement of innovative technologies such as an Intelligent Reflecting surface Aided Wireless network can be further instrumental in reducing the latency of the edge computing [53]. Figure 7 summarizes the various benefits and key points of consideration to be focussed in next few years for the adoption and establishment of MII and digital metrology that shall lead to cost reduction, product standardization, and shall strengthen the digital quality infrastructure in any country.

The inclusive and sustainable industrial development and digitization of quality infrastructure (metrology, standardization and accreditation) shall be complementary to 
Fig. 7 Benefits of digital transformation and propose metrology cloud on the sustainable industrial development in the next decade

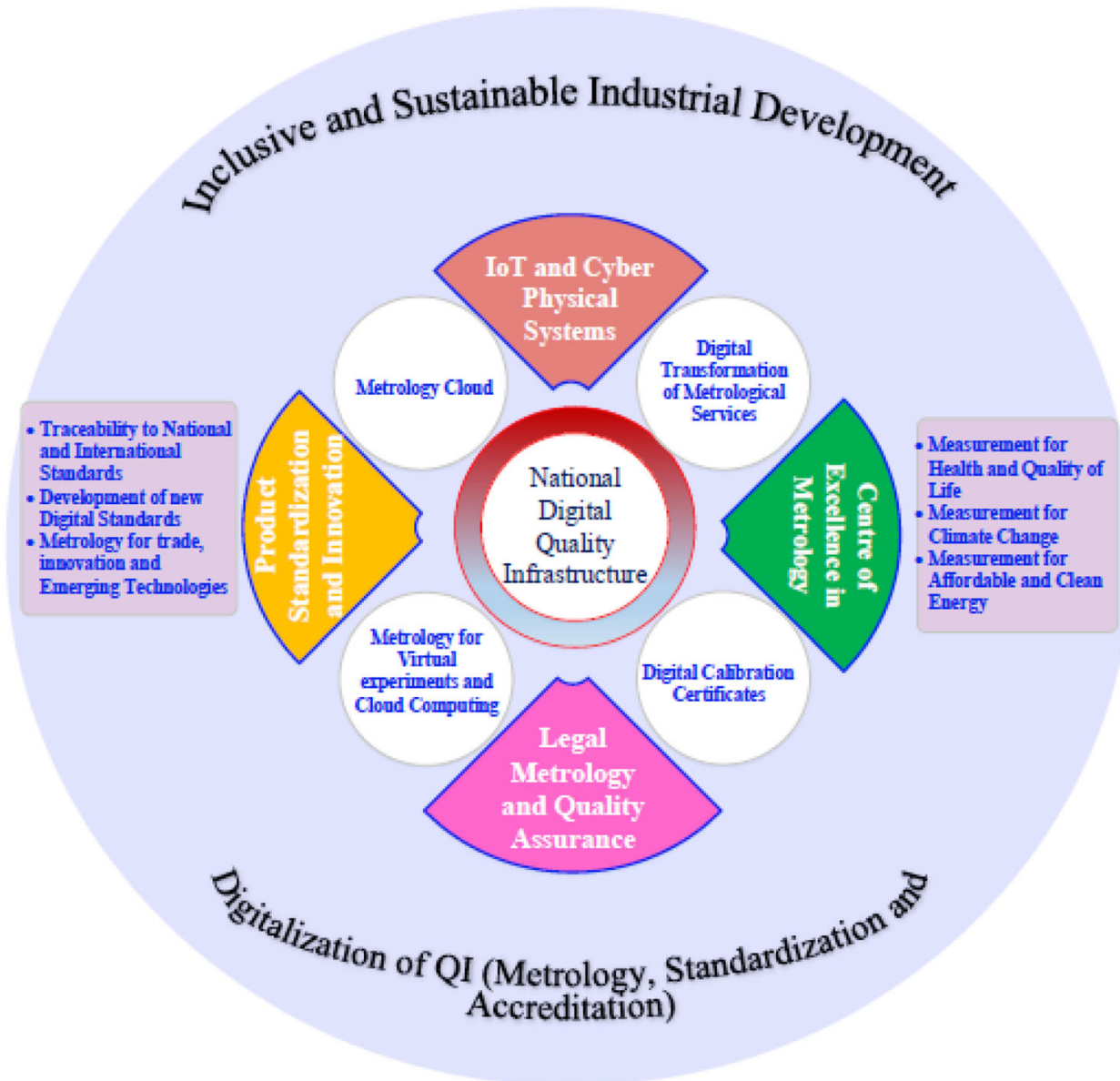

each other and together contribute to the economic and industrial growth. The strengthening of metrology by establishment of quantum standards and quantum-based measurement technologies shall foster the improvement of traceability chain and shall enable precision measurements referenced to international system of units (SI) in industry, academic institutes, avionics, automobile, defence and space research, railways, banking, agriculture and medical sector [54]. The metrology for health and quality of life, climate change, affordable and clean energy has also to be focussed to cater to the growing needs of the population and providing safe and healthy environment for sustainable development goals.

\section{Challenges and The-Way-Ahead}

\subsection{Challenges in Sensor Technologies}

The design and fabrication of smart sensors have thus emerged as a leading research area for researchers in recent times. There is need of more and more accurate and precise measurements that lead to the reliable and robust design of smart sensors. Presently, sensors are being used to record and analyse the data from the most hostile and challenging environment like biomedical measurements, space applications, meteorology applications, physical and chemical metrology, electrical and electronic measurements. Sensors facilitate the condition monitoring of the machines, devices or structures wherein they are integrated. In spite of integrated sensor networks, wireless sensor network has longterm potential and importance, responsible for enhanced throughput based on the collected data. Wireless sensor networks face many conceptual and optimization problem like tracking, deployment and reliability-related issues. Wireless sensors are generally deployed in the harsh environment. Therefore, there may be faulty or unreliable communication with these sensors. The probability of signal delay and loss is common in wireless signal network. The very basic problem with the wireless sensors is the coverage-related issue, which affects the quality of service of the sensor. The major problems that are associated with the wireless sensor network are (i) selection of hardware and operating infrastructure, (ii) calibration, deployment and programming model for the sensing network and (iii) synchronization [34]. Thus, it is imperative to counter these 
limitations associated with the wireless sensors for developing a reliable, economical and usable network that can be used in Industry 4.0 without any hesitation.

\subsection{Challenges in Wireless Communication}

Wireless sensor and communications are the prerequisites for Industry 4.0. However, there are many challenges faced in order to provide a secure communication, which is not prone to attacks. One of the attacks is denial distribution of services. An innovative method to detect attacks on software-defined wireless sensor network is explained in reference [54]. Wormhole is another threat to wireless sensors where an attacker introduces dangerous nodes and uses false routes established by the node. Hence, the route from the sensor is set up and the wormhole is detected using a MAC centralized routing protocol (MCRP) [55]. The blockchain methodology suggested in reference [56] is used to keep the communication links in Industry 4.0 secure. Industry 4.0 is required to have reliable, low latency and energy-efficient communication to allow machine-to-machine communication [57]. 5G provides the communication support for Industry 4.0 and allows the prolonged battery life. In $5 \mathrm{G}$, there is an issue of resource allocation for the network user, which is the wireless sensor. Hence, network slicing is required to distribute the network to the $\mathrm{N}$ sensors [58]. In an industry, there may be more than one sensor using the same network to send information. This increases the traffic in the network, which also causes the loss of data. These lost data must be retransmitted efficiently [59]. The signal from the sensors in a distributed system in Industry may be mixed since the information is passed through air. So, a method of using dense convolution and federal learning is efficient to separate the signal [60].

\subsection{Challenges of Digitalization}

Even though Industry 4.0 has many benefits, one of the requirements for digitalization is sharing of data but most of the companies are reluctant to share data as they are afraid of fall behind their competitors or being exploited by others $[61,62]$. The digitalization possesses risks to the sensitive information, and privacy is being violated as the information is transparent [63]. It infuses fear and lack of trust to adopt digital technologies by industries. In other words, the digital world should provide transparency of data with adequate security. The cloud services face many threats so that personnel with adequate knowledge of how to deal with this threat is necessary to use these services [64, 65]. Other challenges for the Industry 4.0 are considered to be lack of trained employees, lack of competencies, lack of financial resources, lack of standards and processes and resistance from the employees [66]. These aspects have to be resolved step by step for synergising the complete process and establishment of a digital quality infrastructure in Indian perspectives. The trained manpower and experts from various fields have to play a key role in creating awareness and inculcating knowledge about various technical and legal considerations for the establishment of a foolproof system.

\section{Conclusions and Outlook}

The paper highlighted the various aspects pertaining to the smart sensors and IoT-enabled industrial infrastructure that is pivotal for adapting to the advanced technologies based on fusion of smart sensors traceable to digital metrology, traceability and certification to support the Industry 4.0, Smart cities, Digital India and AtmaNirbhar Bharat missions of the government of India. The work presented in this paper discusses about the various challenges associated with the digital transformation of metrology along with the inclusion of smart sensors in Industry 4.0. The work illustrates the concept of digital transformation of metrological applications by implementing intelligent techniques for manufacturing processes along with the development and successful implementation of digital quality infrastructure in India. The present paper is an attempt to provide terse review and national and international perspectives related to the advanced technological developments in this field and the challenges therein. COVID-19 has become an invisible catalyst for digitalization. The pandemic has enlightened the society about the importance and inevitability of the digitalization. The ease with which the work could be monitored and controlled even from the home evoked the interests of the industrialists as well as employees and increased the pace towards digitalization. But still the pace with which the security is provided to the data stored does not match pace of digitalization. The communication issues are still prominent in different corners of the world. For the uninterrupted communication of various IoT devices, a better communication protocol must be introduced. The emergence of $5 \mathrm{G}$ promises the reliability and latency demanded by Industry 4.0. But most of the countries, including India, are in the initial stages of $5 \mathrm{G}$ communication. Thus, in metrology field, the concept of proposed metrology cloud, digital calibration certificate and development of Measurement Information Infrastructure (MII) as that focussed in Europe shall serve as strong pillars for the establishment of a trustworthy national digital quality infrastructure and strengthening the metrology, standardization and accreditation in Indian perspectives. Also, India being the largest exporter of the IT, the merging of Indian industries with the Indian IT infrastructure, 
provides a strong basement for digitalization. This provides a win-win situation to both IT and other sectors to expand the field of research and development. Thus, the integration of various technological advancements in conjunction with IoT shall pave the way for research and development in the field of smart sensors, wireless protocols, IoT devices and cloud computing for Industry 4.0 and shall serve as the backbone for the inclusive growth of industry and ramping up the economy of any country.

\section{References}

[1] M. Wollschlaeger, T. Sauter, J. Jasperneite, The future of industrial communication: automation networks in the era of the internet of things and industry 4.0, IEEE Industrial electronics magazine, 2017;11(1):17-27.

[2] Marcin B. Bauza, Jason Tenboer, Ming Li, Aleksandr Lisovich, Jian Zhou, Daniel Pratt, Jonathan Edwards, Hai Zhang, Charles Turch and Richard Knebel, Realization of industry 4.0 with high-speed CT in high volume production. CIRP Journal of Manufacturing Science and Technology, 22 (2018) 121-125.

[3] Victor Alonso, Angel Dacal-Nieto, LuísBarreto, AntónioAmaral, Eduardo Rivero, Industry 4.0 implications in machine vision metrology: an overview, Procedia Manufacturing 2019, 41, 359-366.

[4] What is Industry 4.0- the Industrial Internet of Things (IIoT), Available from: https://www.epicor.com/en-in/resource-center/ articles/what-is-industry-4-0/.

[5] Roberto Benitez Mr., and Roberto Benitez Jr., Wireless calibration for Industry 4.0, International Congress of Metrology, 2019.

[6] B. Afzal, M. Umair, G.A. Shah and E. Ahmed, Enabling IoT platforms for social IoT applications: Vision, feature mapping, and challenges. Future Gener. Comput. Syst., 92 (2019) 718-731.

[7] Industry 4.0 Building Connected Digital Manufacturing, Available from: https://blog.rgbsi.com/industry-4.0-connected-digital -manufacturing.

[8] Benitez, G.B.; Ayala, N.F.; Frank, A.G., Industry 4.0 innovation ecosystems: An evolutionary perspective on value cocreation. Int. J. Prod. Econ. 2020, 228, 107735.

[9] Goldstein B, NIST on a chip: Revolutionizing Metrology through Deployable, Quantum-Based Sensors, SMSI 2020 Conference-Sensor and Measurement Science International, 30-31.

[10] G. D’Emilia, A. Gaspari, E. Natale, Measurements for smart manufacturing in an Industry 4.0 scenario, Proc. of IEEE International Workshop on Metrology for Industry 4.0 and IoT, Brescia, Italy, April 16-18, 2018, pp. 108-112.

[11] Agostini, L.; Filippini, R. Organizational and managerial challenges in the path toward Industry 4.0. Eur. J. Innov. Manag. 2019, 22, 406-421.

[12] D.K. Aswal, Quality infrastructure of India and its importance for inclusive national growth. MAPAN-J. Metrol. Soc. India, 35 (2020) 139.

[13] D. K. Aswal, Introduction: Metrology for All People for All Time, In: Aswal D.K. (eds) Metrology for Inclusive Growth of India. Springer, Singapore, pp 1-36, https://doi.org/ https://doi.org/10.1007/978-981-15-8872-3_1.

[14] D. K. Aswal, (Ed.), Metrology for Inclusive Growth of India, Springer, Singapore, 2020, https://www.springer.com/gp/book/ 9789811588716.
[15] Metrology for the Factory of the Future, Available from: https://www.euramet.org/research-innovation/search-researchprojects/details/project/metrology-for-the-factory-of-the-future/ ?tx_eurametctcp_project $\% 5 \mathrm{Baction} \% 5 \mathrm{D}=$ show\&tx_eurametct cp_project $\% 5 \mathrm{Bcontroller} \% 5 \mathrm{D}=$ Project $\& \mathrm{~L}=0 \& \mathrm{cHash}=575 \mathrm{fbce}$ 9df841dc03cd08e8cc3099fff

[16] Schütze, N. Helwig, and T. Schneider, Sensors 4.0 - smart sensors and measurement technology enable Industry 4.0, J. Sens. Sens. Syst., 2018, 7, 359-371.

[17] D. Gupta, V. H. C de Albuquerque, A. Khanna and P. L. Mehta, Smart Sensors for Industrial Internet of Things, Springer International Publishing, 2021.

[18] H. C. Mueller, A. Hennig, G. v. Boegel and A. Grabmaier, Wireless Sensors for Industry 4.0 - Wireless Communication and Wireless Powering, Smart SysTech 2018; European Conference on Smart Objects, Systems and Technologies, Munich, Germany, 2018, pp. 1-4.

[19] Chee-Yee Chong and S. P. Kumar, Sensor networks: evolution, opportunities, and challenges, Proceedings of the IEEE, vol. 91, no. 8, Aug. 2003, pp. 1247-1256.

[20] T. Mustapää, J. Autiosalo, P. Nikander, J. E. Siegel and R. Viitala, "Digital Metrology for the Internet of Things," 2020 Global Internet of Things Summit (GIoTS), Dublin, Ireland, 2020, pp. 1-6.

[21] Metrology for the digitalization of the economy and society, Available from: https://www.bipm.org/cc/PARTNERS/Allo wed/2017_October/2017-Metrology-for-the-Digitalisation-ofEconomy-and-Society.pdf

[22] Thiel, Florian, Marko Esche, Federico Grasso Toro, Alexander Oppermann, Jan Wetzlich, and Daniel Peters. "The European metrology cloud." In $18^{\text {th }}$ International Congress of Metrology, p. 09001. EDP Sciences, 2017.

[23] What is Industry 4.0? Available from: https://www.ibm. com/topics/industry-4-0

[24] M. S. Bhatia and S. Kumar, Critical Success Factors of Industry 4.0 in Automotive Manufacturing Industry, IEEE Transactions on Engineering Management, 2020.

[25] S. Fernández-Miranda, M. Marcos, M. Peralta, F. Aguayo, The challenge of integrating Industry 4.0 in the degree of Mechanical Engineering. Procedia Manufacturing. 2017, 13, 1229-1236.

[26] Wilkesmann, M.; Wilkesmann, U. Industry 4.0 —organizing routines or innovations? VINE J. Inf. Knowl. Manag. Syst. 2018, 48, 238-254.

[27] L. Barreto, A. Amaral, T. Pereira, Industry 4.0 implications in logistics: an overview, Procedia Manufacturing, 2017;13: $1245-1252$.

[28] Kiel, Daniel, Julian M. Müller, Christian Arnold, and Kai-Ingo Voigt. "Sustainable industrial value creation: Benefits and challenges of industry 4.0." International journal of innovation management 21, no. 08 (2017): 1740015.

[29] Tortorella, G.L.; Giglio, R.; Van Dun, D.H. Industry 4.0 adoption as a moderator of the impact of lean production practices on operational performance improvement. Int. J. Oper. Prod. Manag. 2019, 39, 860-886.

[30] Leng, J.; Ruan, G.; Jiang, P.; Xu, K.; Liu, Q.; Zhou, X.; Liu, C. Blockchain-empowered sustainable manufacturing and product lifecycle management in industry 4.0: A survey. Renew. Sustain. Energy Rev. 2020, 132, 110112.

[31] Guideline Sensors for Industry 4.0, Available from: https://industrie40.vdma.org/en/viewer/-/v2article/render/25266 556 .

[32] G. D’Emilia, A. Gaspari, Data validation technics for measurements systems operating in an Industry 4.0 scenario, Proc. of IEEE International Workshop on Metrology for Industry 4.0 and IoT, Brescia, Italy, April 16-18, 2018, pp. 113-117. 
[33] Kalsoom, T.; Ramzan, N.; Ahmed, S.; Ur-Rehman, M. Advances in Sensor Technologies in the Era of Smart Factory and Industry 4.0. Sensors 2020, 20, 6783.

[34] Vidosav Majstorovic, Slavenko Stojadinovic, Srdjan Zivkovic, Dragan Djurdjanovic, Zivana Jakovljevic and Nemanja Gligorijevic, Cyber-physical manufacturing metrology model (CPM3) for sculptured surfaces-turbine blade application. Procedia CIRP, 63 (2017) 658-663.

[35] R. Benitez, R. Benitez, C. Ramirez and J. A. Vazquez, Sensors calibration for Metrology 4.0, II Workshop on Metrology for Industry 4.0 and IoT (MetroInd4.0\&IoT), Naples, Italy, 2019, pp. 296-299.

[36] http://statisticstimes.com/economy/country/india-gdp-sectorwise. $\mathrm{php}$

[37] https://www.mckinsey.com/business-functions/mckinsey-digital /our-insights/digital-india-technology-to-transform-a-connected -nation\#

[38] Castelo-Branco, I.; Cruz-Jesus, F.; Oliveira, T. Assessing Industry 4.0 readiness in manufacturing: Evidence forthe European Union. Comput. Ind. 2019, 107, 22-32..

[39] M. Compare, P. Baraldi and E. Zio, Challenges to IoT-Enabled Predictive Maintenance for Industry 4.0, IEEE Internet of Things Journal, 7, 5, 4585-4597, 2020.

[40] D.K. Aswal, Quality infrastructure of India and its importance for inclusive national growth. MAPAN, 35 (2020) 139-150.

[41] S. Andonov, M. Cundeva-Blajer, Calibration for Industry 4.0 Metrology: Touchless Calibration, Journal of Physics: Conference Series, 2018;1065:072019.

[42] R. Taymanov, K. Sapozhnikova, Metrology challenges of Industry 4.0, Journal of Physics: Conference Series, 2018;1065:072044.

[43] Kaya, M. Cagri, Mahdi Saeedi Nikoo, Michael L. Schwartz, and Halit Oguztuzun. "Internet of measurement things architecture: Proof of concept with scope of accreditation." Sensors 20, no. 2 (2020): 503.

[44] Kuster M J, Toward a measurement information infrastructure, Metrologist, vol. 6-pres no., Jan 2013

[45] Hackel H G, Härtig F, Hornig J, Wiedenhöfer T, The digital calibration certificate, in Metrology for the Digitalization of the Economy and Society, PTB, Germany, Dec. 2017, 127, no. 4, 75-81.

[46] Forging the digital twin in discrete manufacturing: a vision for unity in the virtual and real worlds. https://www.lnsresearch.com/ research-library/research-articles/ebook-forging-the-digital-twinin-discrete-manufacturing-a-vision-for-unity-in-the-virtual-andreal-worlds.

[47] Louise Wright and Stuart Davidson, How to tell the difference between a model and a digital twin. Advanced Modeling and Simulation in Engineering Sciences, 7 (2020) 1-13.

[48] F.L.O.R.I.A.N. Thiel, Digital transformation of legal metrologythe European metrology cloud. OIML Bulletin, 59 (2018) $10-21$.

[49] Ghobakhloo, Morteza. "Industry 4.0, digitization, and opportunities for sustainability." Journal of Cleaner Production 252 (2020): 119869

[50] Alessia Napoleone, Marco Macchi and Alessandro Pozzetti, A review on the characteristics of cyber-physical systems for the future smart factories. Journal of manufacturing systems, 54 (2020) 305-335.

[51] Compare, Michele, PieroBaraldi, and Enrico Zio. "Challenges to IoT-enabled predictive maintenance for industry 4.0." IEEE Internet of Things Journal 7.5 (2019): 4585-4597.
[52] Ouns Bouachir, Moayad Aloqaily, Lewis Tseng and Azzedine Boukerche, Blockchain and fog computing for cyberphysical systems: The case of smart industry. Computer, 53 (2020) 36-45.

[53] Tong Bai, Cunhua Pan, Yansha Deng, Maged Elkashlan, Arumugam Nallanathan and Lajos Hanzo, Latency minimization for intelligent reflecting surface aided mobile edge computing. IEEE Journal on Selected Areas in Communications, 38 (2020) 2666-2682.

[54] Segura, Gustavo A. Nunez, Sotiris Skaperas, Arsenia Chorti, Lefteris Mamatas, and Cintia Borges Margi. "Denial of Service Attacks Detection in Software-Defined Wireless Sensor Networks." In 2020 IEEE International Conference on Communications Workshops (ICC Workshops), pp. 1-7. IEEE, 2020.

[55] OhidaRufai Ahutu and Hosam El-Ocla, Centralized Routing Protocol for Detecting Wormhole Attacks in Wireless Sensor Networks. IEEE Access, 8 (2020) 63270-63282.

[56] Rathee, Geetanjali, M. Balasaraswathi, K. Prabhu Chandran, Sharmi Dev Gupta, and C. S. Boopathi. "A secure IoT sensors communication in industry 4.0 using blockchain technology." Journal of Ambient Intelligence and Humanized Computing (2020): 1-13

[57] Da Costa, Daniel Benevides, and Hong-Chuan Yang. "Grand Challenges in Wireless Communications." Frontiers in Communications and Networks 1 (2020): 1.

[58] Fossati, Francesca, Stefano Moretti, Stephane Rovedakis, and Stefano Secci. "Decentralization of $5 \mathrm{G}$ slice resource allocation." In NOMS 2020-2020 IEEE/IFIP Network Operations and Management Symposium, pp. 1-9. IEEE, 2020

[59] Bulashenko, Andrew, Stepan Piltyay, Alina Polishchuk, and Oleksandr Bulashenko. "New traffic model of M2M technology in 5G wireless sensor networks." In 2020 IEEE 2nd International Conference on Advanced Trends in Information Theory (ATIT), pp. 125-131. IEEE, 2020

[60] Liu, Mingqian, Ke Yang, Nan Zhao, Yunfei Chen, Hao Song, and Fengkui Gong. "Intelligent Signal Classification in Industrial Distributed Wireless Sensor Networks-Based IIoT.” IEEE Transactions on Industrial Informatics (2020).

[61] Raphael Preindl, KonstantinosNikolopoulos\&KonstantiaLitsiou (2020) Transformation strategies for the supply chain: the impact of industry 4.0 and digital transformation, Supply Chain Forum: An International Journal, 21:1, 26-34, DOI: https://doi.org/10.1080/16258312.2020.1716633

[62] Aminzoui, Abla, and Joacim Knapp. "Validation and Ranking of Challenges in Digital Transformation towards Industry 4.0: a multi-case study in Swedish manufacturing SMEs." (2020).

[63] Culot, Giovanna, Guido Nassimbeni, Guido Orzes, and Marco Sartor. "Behind the definition of Industry 4.0: Analysis and open questions." International Journal of Production Economics (2020): 107617

[64] Tabrizchi, Hamed, and MarjanKuchaki Rafsanjani. "A survey on security challenges in cloud computing: issues, threats, and solutions." The journal of supercomputing 76.12 (2020): 9493-9532

[65] Abdul-Hamid, Asma-Qamaliah, Mohd Helmi Ali, Ming-Lang Tseng, Shulin Lan, and Mukesh Kumar. "Impeding challenges on industry 4.0 in circular economy: Palm oil industry in Malaysia." Computers \& Operations Research 123 (2020): 105052.

[66] Vuksanović Herceg, Iva, Vukašin Kuč, Veljko M. Mijušković, and Tomislav Herceg. "Challenges and driving forces for industry 4.0 implementation." Sustainability 12, no. 10 (2020): 4208.

Publisher's Note Springer Nature remains neutral with regard to jurisdictional claims in published maps and institutional affiliations. 